\title{
Localization in finite asymmetric vibro-impact chains
}

\author{
Itay Grinberg ${ }^{1, *}$ and Oleg V. Gendelman ${ }^{1, \dagger}$ \\ ${ }^{1}$ Faculty of Mechanical Engineering \\ Technion - Israel Institute of Technology
}

(Dated: March 6, 2022)

\begin{abstract}
We explore the dynamics of strongly localized periodic solutions (discrete solitons, or discrete breathers) in a finite one-dimensional chain of asymmetric vibro-impact oscillators. The model involves a parabolic on-site potential with asymmetric rigid constraints (the displacement domain of each particle is finite), and a linear nearest-neighbor coupling. When the particle approaches the constraint, it undergoes an impact (not necessarily elastic), that satisfies Newton impact law. Nonlinearity of the system stems from the impacts; their possible non-elasticity is the sole source of damping in the system. We demonstrate that this vibro-impact model allows derivation of exact analytic solutions for the asymmetric discrete breathers, both in conservative and forced-damped settings. The asymmetry makes two types of breathers possible: breathers that impact both or only one constraint. Transition between these two types of the breathers corresponds to a grazing bifurcation. Special character of the nonlinearity permits explicit derivation of a monodromy matrix. Therefore, the stability of the obtained breather solutions can be exactly studied in the framework of simple methods of linear algebra, and with rather moderate computational efforts. All three generic scenarios of the loss of stability (pitchfork, Neimark-Sacker and period doubling bifurcations) are observed.
\end{abstract}

PACS numbers: 05.45.Yv, 63.20.Pw, 63.20.Ry

\section{INTRODUCTION}

Localization is an important and widely studied phenomenon in discrete dynamical systems [1 9]. Contrary to the localization in linear systems, in nonlinear systems it is possible without any disorder, i.e. localization may occur even in a purely homogeneous nonlinear lattice. Interesting examples of such localized responses are Discrete Breathers (DBs), sometimes referred to as Intrinsic Localized Modes (ILMs) or Discrete Solitons. The DB is a periodic strongly localized response of the lattice system. It can be simply imagined as an oscillating envelope localized in the vicinity of a single or several sites of the lattice. The DB's localization is typically exponential; however, in the systems with strong nonlinearity, it may be hyper-exponential[2]. The DBs are known in various branches in physics. They were experimentally observed and theoretically discussed in a variety of model systems, such as superconducting Josephson junctions [10], nonlinear magnetic metamaterials[11, electrical lattices [12, micro-mechanical cantilever arrays 13 17, Bose-Einstein condensates 18, and chains of mechanical oscillators 1923.

The exact DB solutions in specific nonlinear chain models remain scarce due to the nonlinearity and discreteness of the systems that encumbers the derivation of exact solutions. Current theoretical research primarily concentrates on numerical explorations and approximate analytic approaches 1, 2, 9, 24]. Few known exceptions are the completely integrable Ablowitz-Ladic model 25],

\footnotetext{
* GrinbergItay@gmail.com

$\dagger$ ovgend@technion.ac.il
}

chains with homogeneous interaction[26], and vibroimpact chains 19. Recently the latter approach was extended to the forced-damped vibro-impact chains 21, 23, chains with self-excitation 27, and, most recently, to Multi-Breather (MB) solutions [22], namely the DBs with more than a single localization site.

The aforementioned vibro-impact chains are essentially linear, except for the possibility of collisions, i.e. all the on-site and coupling interactions, that are not impacts, are linear. This feature not only allows the derivation of the exact solution, but also considerably simplifies the stability analysis. The stability of the periodic DB solution is determined by location of the eigenvalues of the Monodromy matrix [28]. In most cases, the monodromy matrix can only by obtained numerically by integration of the equations of motion over the period of the solution. This task can be extremely difficult when treating systems with a large number of particles, to the extent that super-computers may be necessary. The considered vibro-impact models allow explicit derivation of the monodromy matrix $21-23$. Thus the stability analysis is reduced to evaluation of the spectrum of easily computed matrices. Consequently, even simple PCs suffice for chains with thousands of particles. Furthermore, even for the finite chains, one can accomplish these derivations without further approximations.

This work is based on the approach used in refs. [19, 21 23], but introduces important novel feature into the model. In symmetric models, a breakdown of the DB symmetry is one of the instability scenarios [21. In current model, the asymmetry is imbedded into the lattice itself. To be more specific, the model comprises a finite number of linearly coupled oscillators; each of the latter includes on-site coupling with two asymmetric rigid barriers bounding the movement. Thus one can obtain the 
asymmetric DBs and explore their zones of existence and stability properties in the space of parameters. Furthermore, the considered asymmetry allows a new type of the DB - the single-sided DB - where the impact only occurs at one of the constraints. In sec. I we present a detailed description of the system. For this new type of DBs we observe for the first time the period doubling bifurcations and obtain an analytic solution for the emerging solution in a similar manner. Section [1] contains the derivations of an exact analytic solution for both conservative and forced/damped DBs, as well as for the single-sided DB. The method of the stability analysis is explained in sec. III. Numerical validation of the results is presented in sec. IV, followed by concluding remarks in sec. V.

\section{DESCRIPTION OF THE MODEL}

We consider a chain of $(N+1)$ identical unit masses, coupled to their neighbors via linear springs, and with periodic boundary conditions. In addition, all masses are subject to identical on-site potentials. The on-site interaction is via linear spring, but the motion is bounded by a set of asymmetric impact barriers. The on-site and coupling potentials can be described as follows:

$$
\begin{gathered}
V(u)= \begin{cases}\gamma_{1} u^{2} & |u-a|<1 \\
\text { Impact } & |x-a|=1\end{cases} \\
W(u)=\gamma_{2} u^{2}
\end{gathered}
$$

where $a \geq 0$ is the parameter of asymmetry and $\gamma_{1}$ and $\gamma_{2}$ are the on-site and coupling stiffnesses, respectively.

This yields the following Hamiltonian for the full finite chain:

$$
\begin{gathered}
H=\sum_{n=0}^{N}\left(\frac{1}{2} p_{n}^{2}+V\left(u_{n}\right)\right)+ \\
+\sum_{n=0}^{N-1} W\left(u_{n}-u_{n+1}\right)+W\left(u_{N}-u_{0}\right)
\end{gathered}
$$

The impact, which could be either elastic or nonelastic, obeys the following traditional Newton impact law:

$$
\dot{u}\left(t_{i}+\right)=-e \dot{u}\left(t_{i}-\right)
$$

where $t_{i}$ is the time instance of the impact and $0<e \leq 1$ is the coefficient of restitution.

\section{EXACT SOLUTION FOR THE ASYMMETRIC BREATHER}

\section{A. Discrete Breathers in the Conservative Model}

If the external forcing is absent, one should set the coefficient of restitution to $e=1$ in order to preclude the dissipation. Equations of motion for the chain can be easily obtained from the Hamiltonian given in (3). Furthermore, the periodicity of the DB allows introducing the impact into the equations of motion as external forcing in the the form of a sum of delta functions with advanced-delayed arguments. Without restricting the generality, we adopt that the DB is localized at the particle with $n=0$. One obtains the following equations of motion:

$$
\begin{gathered}
\ddot{u}_{0}+\gamma_{1} u_{0}+\gamma_{2}\left(2 u_{0}-u_{1}-u_{N}\right)= \\
=2 p_{1} \sum_{j=-\infty}^{\infty} \delta\left(t-\phi-\frac{2 \pi j}{\omega}\right)- \\
-2 p_{2} \sum_{j=-\infty}^{\infty} \delta\left(t-\frac{2 \pi j}{\omega}\right) \\
\ddot{u}_{n}+\gamma_{1} u_{n}+\gamma_{2}\left(2 u_{n}-u_{n+1}-u_{n-1}\right)=0 \\
\ddot{u}_{N}+\gamma_{1} u_{N}+\gamma_{2}\left(2 u_{N}-u_{0}-u_{N-1}\right)=0
\end{gathered}
$$

where $2 p_{1}$ and $2 p_{2}$ correspond to the amounts of momentum transferred in the course of each of the impacts, $\delta$ is the Dirac delta and $\phi$ is the phase instance of the secondary impact in the period of the DB.

The expression for the impact forcing can be re-written in the form of generalized Fourier series:

$$
\begin{gathered}
\ddot{u}_{0}+\gamma_{1} u_{0}+\gamma_{2}\left(2 u_{0}-u_{1}-u_{N}\right)= \\
=\frac{\omega}{\pi} \sum_{j=-\infty}^{\infty}\left(p_{1} \cos (j \omega(t-\phi))-p_{2} \cos (j \omega t)\right) \\
\ddot{u}_{n}+\gamma_{1} u_{n}+\gamma_{2}\left(2 u_{n}-u_{n+1}-u_{n-1}\right)=0 \\
\ddot{u}_{N}+\gamma_{1} u_{N}+\gamma_{2}\left(2 u_{N}-u_{0}-u_{N-1}\right)=0
\end{gathered}
$$

Thus, one further obtains:

$$
\begin{gathered}
\ddot{u}_{0}+\gamma_{1} u_{0}+\gamma_{2}\left(2 u_{0}-u_{1}-u_{N}\right)=\frac{\omega}{\pi}\left(p_{1}-p_{2}\right)+ \\
+\frac{2 \omega}{\pi} \sum_{j=1}^{\infty}\left(p_{1} \cos (j \omega(t-\phi))-p_{2} \cos (j \omega t)\right) \\
\ddot{u}_{n}+\gamma_{1} u_{n}+\gamma_{2}\left(2 u_{n}-u_{n+1}-u_{n-1}\right)=0 \\
\ddot{u}_{N}+\gamma_{1} u_{N}+\gamma_{2}\left(2 u_{N}-u_{0}-u_{N-1}\right)=0
\end{gathered}
$$

To obtain the exact solution, the displacement of each particle is also searched in the form of Fourier series, and the following anzats is used:

$$
u_{n}=u_{n, 0}+\sum_{j=1}^{\infty}\left(u_{n, j, 1} \cos (j \omega(t-\phi))+u_{n, j, 2} \cos (j \omega t)\right)
$$

where,

$$
\begin{aligned}
u_{n, 0} & =A_{0} f_{0}^{n}+B_{0} f_{0}^{-n} \\
u_{n, j, 1} & =A_{j} f_{j}^{n}+B_{j} f_{j}^{-n} \\
u_{n, j, 2} & =C_{j} f_{j}^{n}+D_{j} f_{j}^{-n}
\end{aligned}
$$


Linearity of the equations of motion between the impacts yields:

$$
\begin{aligned}
& f_{j}=\frac{\gamma_{1}+2 \gamma_{2}-j^{2} \omega^{2} \pm \sqrt{\left(j^{2} \omega^{2}-\gamma_{1}-2 \gamma_{2}\right)^{2}-4 \gamma_{2}^{2}}}{2 \gamma_{2}}= \\
& =\frac{\gamma_{1}+2 \gamma_{2}-j^{2} \omega^{2} \pm \sqrt{\left(j^{2} \omega^{2}-\gamma_{1}-4 \gamma_{2}\right)\left(j^{2} \omega^{2}-\gamma_{1}\right)}}{2 \gamma_{2}}
\end{aligned}
$$

Account of the periodic boundary conditions in eq. 13 yields the following relations:

$$
\begin{aligned}
& A_{j}=B_{j} f_{j}^{-N-1} \\
& C_{j}=D_{j} f_{j}^{-N-1}
\end{aligned}
$$

Finally, substituting these derivations into the first equation of system (11), that describes the dynamics of impacting mass, one obtains for $j>0$ :

$$
\begin{aligned}
& B_{j}=-\frac{2 \omega p_{1}}{\pi \gamma_{2}\left(f_{j}-f_{j}^{-1}\right)\left(f_{j}^{-N-1}-1\right)} \\
& D_{j}=\frac{2 \omega p_{2}}{\pi \gamma_{2}\left(f_{j}-f_{j}^{-1}\right)\left(f_{j}^{-N-1}-1\right)}
\end{aligned}
$$

and for $j=0$ :

$$
B_{0}=\frac{\omega\left(p_{2}-p_{1}\right)}{\pi \gamma_{2}\left(f_{0}-f_{0}^{-1}\right)\left(f_{0}^{-N-1}-1\right)}
$$

Summarizing, one obtains the following exact solution for the DB:

$u_{n}=u_{n, 0}+\sum_{j=1}^{\infty}\left(u_{n, j, 1} \cos (j \omega(t-\phi))+u_{n, j, 2} \cos (j \omega t)\right)$

where

$$
\begin{aligned}
u_{n, 0} & =\frac{\omega\left(p_{2}-p_{1}\right)\left(f_{0}^{n-N-1}+f_{0}^{-n}\right)}{\pi \gamma_{2}\left(f_{0}-f_{0}^{-1}\right)\left(f_{0}^{-N-1}-1\right)} \\
u_{n, j, 1} & =-\frac{2 \omega p_{1}\left(f_{j}^{n-N-1}+f_{j}^{-n}\right)}{\pi \gamma_{2}\left(f_{j}-f_{j}^{-1}\right)\left(f_{j}^{-N-1}-1\right)} \\
u_{n, j, 2} & =\frac{2 \omega p_{2}\left(f_{j}^{n-N-1}+f_{j}^{-n}\right)}{\pi \gamma_{2}\left(f_{j}-f_{j}^{-1}\right)\left(f_{j}^{-N-1}-1\right)}
\end{aligned}
$$

To obtain the values of the unknown parameters, we explicitly take into account the conditions of impacts, that should be enforced when the particle achieves the barriers:

$$
\begin{gathered}
u_{0}(0)=\frac{\omega\left(f_{0}^{-N-1}+1\right)\left(p_{2}-p_{1}\right)}{\pi \gamma_{2}\left(f_{0}-f_{0}^{-1}\right)\left(f_{0}^{-N-1}-1\right)}- \\
-\sum_{j=1}^{\infty} \frac{2 \omega\left(f_{j}^{-N-1}+1\right) p_{1}}{\pi \gamma_{2}\left(f_{j}-f_{j}^{-1}\right)\left(f_{j}^{-N-1}-1\right)} \cos (j \omega \phi)+ \\
+\sum_{j=1}^{\infty} \frac{2 \omega\left(f_{j}^{-N-1}+1\right) p_{2}}{\pi \gamma_{2}\left(f_{j}-f_{j}^{-1}\right)\left(f_{j}^{-N-1}-1\right)}=1+a
\end{gathered}
$$

$$
\begin{gathered}
u_{0}(\phi)=\frac{\omega\left(f_{0}^{-N-1}+1_{0}\right)\left(p_{2}-p_{1}\right)}{\pi \gamma_{2}\left(f_{0}-f_{0}^{-1}\right)\left(f_{0}^{-N-1}-1\right)}- \\
-\sum_{j=1}^{\infty} \frac{2 \omega\left(f_{j}^{-N-1}+1\right) p_{1}}{\pi \gamma_{2}\left(f_{j}-f_{j}^{-1}\right)\left(f_{j}^{-N-1}-1\right)}+ \\
+\sum_{j=1}^{\infty} \frac{2 \omega\left(f_{j}^{-N-1}+1\right) p_{2}}{\pi \gamma_{2}\left(f_{j}-f_{j}^{-1}\right)\left(f_{j}^{-N-1}-1\right)} \cos (j \omega \phi)= \\
=-1+a
\end{gathered}
$$

Reordering these equations, one can write them down in a somewhat simplified form:

$$
\begin{aligned}
-p_{1} \chi_{1}(\phi)+p_{2} \chi_{2} & =1+a \\
-p_{1} \chi_{2}+p_{2} \chi_{1}(\phi) & =-1+a
\end{aligned}
$$

where

$$
\begin{gathered}
\chi_{1}(\phi) \equiv \frac{\omega}{\pi \gamma_{2}}\left(\begin{array}{c}
\frac{\left(f_{0}^{-N-1}+1\right)}{\left(f_{0}-f_{0}^{-1}\right)\left(f_{0}^{-N-1}-1\right)}+ \\
+\sum_{j=1}^{\infty} \frac{2\left(f_{j}^{-N-1}+1\right)}{\left(f_{j}-f_{j}^{-1}\right)\left(f_{j}^{-N-1}-1\right)} \cos (j \omega \phi)
\end{array}\right) \\
\chi_{2} \equiv \frac{\omega}{\pi \gamma_{2}}\left(\begin{array}{c}
\frac{\left(f_{0}^{-N-1}+1\right)}{\left(f_{0}-f_{0}^{-1}\right)\left(f_{0}^{-N-1}-1\right)}+ \\
+\sum_{j=1}^{\infty} \frac{2\left(f_{j}^{-N-1}+1\right)}{\left(f_{j}-f_{j}^{-1}\right)\left(f_{j}^{-N-1}-1\right)}
\end{array}\right)
\end{gathered}
$$

So far there are 3 unknowns $\left(p_{1}, p_{2}\right.$ and $\left.\phi\right)$ and only 2 equations. Additional equation is derived from the condition of energy conservation in the course of each impact:

$$
\dot{u}_{0}(0)=-\omega \sum_{j=1}^{\infty} u_{0, j, 1} j \sin (j \omega \phi)=0
$$

Due to orthogonality of $\sin (j \omega \phi)$, these conditions can hold only for $\phi=\pi / \omega$. In other terms, the DB solution turns out to be symmetric with respect to the time inversion. Fortunately, this conclusion crucially simplifies the problem as the expression for $\chi_{1}$ takes the following form:

$$
\chi_{1}=\frac{\omega}{\pi \gamma_{2}}\left(\begin{array}{c}
\frac{\left(f_{0}^{-N-1}+1\right)}{\left(f_{0}-f_{0}^{-1}\right)\left(f_{0}^{-N-1}-1\right)}+ \\
+\sum_{j=1}^{\infty} \frac{2(-1)^{j}\left(f_{j}^{-N-1}+1\right)}{\left(f_{j}-f_{j}^{-1}\right)\left(f_{j}^{-N-1}-1\right)}
\end{array}\right)
$$

With $\phi$ no longer an unknown, eq. (30)-(31) can now easily be solved:

$$
\begin{aligned}
& p_{1}=\frac{1}{\chi_{2}-\chi_{1}}-\frac{a}{\chi_{1}+\chi_{2}} \\
& p_{2}=\frac{1}{\chi_{2}-\chi_{1}}+\frac{a}{\chi_{1}+\chi_{2}}
\end{aligned}
$$

Summarizing, we obtain the following exact solution 
for the conservative DB:

$$
\begin{gathered}
u_{n}(t)=\frac{4 \omega\left(f_{0}^{n-N-1}+f_{0}^{-n}\right) a}{\pi \gamma_{2}\left(f_{0}-f_{0}^{-1}\right)\left(f_{0}^{-N-1}-1\right)\left(\chi_{1}+\chi_{2}\right)}+ \\
+\sum_{j=1}^{\infty}\left(\frac{8 \omega a\left(f_{2 j}^{n-N-1}+f_{2 j}^{-n}\right)}{\pi \gamma_{2}\left(f_{2 j}-f_{2 j}^{-1}\right)\left(f_{2 j}^{-N-1}-1\right)\left(\chi_{1}+\chi_{2}\right)}\right) \cos (j \omega t)+ \\
+\sum_{j=1}^{\infty}\left(\frac{8 \omega\left(f_{2 j-1}^{n-N-1}+f_{2 j-1}^{-n}\right)}{\pi \gamma_{2}\left(f_{2 j-1}-f_{2 j-1}^{-1}\right)\left(f_{2 j-1}^{-N-1}-1\right)\left(\chi_{2}-\chi_{1}\right)}\right) \cos (j \omega t)
\end{gathered}
$$

\section{Single-Sided Discrete Breathers}

The asymmetric barriers allow a new type of DB solution - the single-sided DB, i.e. the regime, in which the impacting mass does not reach more distant barrier and impacts only one of the barriers. Consequently, this regime can be described by the following equations of motion:

$$
\begin{gathered}
\ddot{u}_{0}+\gamma_{1} u_{0}+\gamma_{2}\left(2 u_{0}-u_{1}-u_{N}\right)=2 p \sum_{j=-\infty}^{\infty} \delta\left(t-\frac{2 \pi j}{\omega}\right) \\
\ddot{u}_{n}+\gamma_{1} u_{n}+\gamma_{2}\left(2 u_{n}-u_{n+1}-u_{n-1}\right)=0 \\
\ddot{u}_{N}+\gamma_{1} u_{N}+\gamma_{2}\left(2 u_{N}-u_{0}-u_{N-1}\right)=0
\end{gathered}
$$

As previously, the system is closed with the help of equations, that fix the impact at the desired location:

$$
u_{0}(0)=-1+a
$$

Finally, the solution is written as follows:

$$
\begin{gathered}
u_{n}=-\frac{\omega(1-a)\left(f_{0}^{n-N-1}+f_{0}^{-n}\right)}{\pi \gamma_{2} \chi_{2}\left(f_{0}-f_{0}^{-1}\right)\left(f_{0}^{-N-1}-1\right)}- \\
-\sum_{j=1}^{\infty} \frac{2 \omega(1-a)\left(f_{j}^{n-N-1}+f_{j}^{-n}\right)}{\pi \gamma_{2} \chi_{2}\left(f_{j}-f_{j}^{-1}\right)\left(f_{j}^{-N-1}-1\right)} \cos (j \omega t)
\end{gathered}
$$

The complete derivation of the solution is available in Appendix A.

\section{B. Discrete Breathers in the Forced-Damped Setting}

Now let us adopt that all masses are subjected to an external force $F(t)$. We examine the case of a symmetric force $F(t)$ which satisfies $F(t)=F(t+2 \pi / \omega)$ and $F(t)=-F(t+\pi / \omega)$. Additionally, the damping is introduced through the non-unit restitution coefficient $0<e<1$. Similarly to the conservative case, we look for the periodic solution, thus the impacts can be introduced in the same form as above. The solution should obey the following set of equations:

$$
\begin{gathered}
\ddot{v}_{0}+\gamma_{1} v_{0}+\gamma_{2}\left(2 v_{0}-v_{1}-v_{N}\right)=F(t+\psi)+ \\
+2 p_{1} \sum_{j=-\infty}^{\infty} \delta\left(t-\phi-\frac{2 \pi j}{\omega}\right)- \\
-2 p_{2} \sum_{j=-\infty}^{\infty} \delta\left(t-\frac{2 \pi j}{\omega}\right) \\
\ddot{v}_{n}+\gamma_{1} v_{n}+\gamma_{2}\left(2 v_{n}-v_{n+1}-v_{n-1}\right)=F(t+\psi) \\
\ddot{v}_{N}+\gamma_{1} v_{N}+\gamma_{2}\left(2 v_{N}-v_{0}-v_{N-1}\right)=F(t+\psi)
\end{gathered}
$$

where $\psi$ is the phase of the external force with respect to DB's impacts.

The external force $F(t)$ can be removed from the equations with the help of a simple transformation. Let $v_{n}(t)=u_{n}(t)+G(t+\psi)$ where $\ddot{G}(t)+\gamma_{1} G(t)=F(t)$. Substitution into the above equations yields:

$$
\begin{gathered}
\ddot{u}_{0}+\gamma_{1} u_{0}+\gamma_{2}\left(2 u_{0}-u_{1}-u_{N}\right)= \\
=2 p_{1} \sum_{j=-\infty}^{\infty} \delta\left(t-\phi-\frac{2 \pi j}{\omega}\right)- \\
-2 p_{2} \sum_{j=-\infty}^{\infty} \delta\left(t-\frac{2 \pi j}{\omega}\right) \\
\ddot{u}_{n}+\gamma_{1} u_{n}+\gamma_{2}\left(2 u_{n}-u_{n+1}-u_{n-1}\right)=0 \\
\ddot{u}_{N}+\gamma_{1} u_{N}+\gamma_{2}\left(2 u_{N}-u_{0}-u_{N-1}\right)=0
\end{gathered}
$$

Similarly, the impact forcing terms are re-written in the form of generalized Fourier series:

$$
\begin{gathered}
\ddot{u}_{0}+\gamma_{1} u_{0}+\gamma_{2}\left(2 u_{0}-u_{1}-u_{N}\right)= \\
=\frac{\omega}{\pi} \sum_{j=-\infty}^{\infty}\left(p_{1} \cos (j \omega(t-\phi))-p_{2} \cos (j \omega t)\right) \\
\ddot{u}_{n}+\gamma_{1} u_{n}+\gamma_{2}\left(2 u_{n}-u_{n+1}-u_{n-1}\right)=0 \\
\ddot{u}_{N}+\gamma_{1} u_{N}+\gamma_{2}\left(2 u_{N}-u_{0}-u_{N-1}\right)=0
\end{gathered}
$$

The equations are identical to those of the conservative model. Hence, the solution is similar:

$$
u_{n}=u_{n, 0}+\sum_{j=1}^{\infty}\left(u_{n, j, 1} \cos (j \omega(t-\phi))+u_{n, j, 2} \cos (j \omega t)\right)
$$

where

$$
\begin{aligned}
u_{n, 0} & =\frac{\omega\left(p_{2}-p_{1}\right)\left(f_{0}^{n-N-1}+f_{0}^{-n}\right)}{\pi \gamma_{2}\left(f_{0}-f_{0}^{-1}\right)\left(f_{0}^{-N-1}-1\right)} \\
u_{n, j, 1} & =-\frac{2 \omega p_{1}\left(f_{j}^{n-N-1}+f_{j}^{-n}\right)}{\pi \gamma_{2}\left(f_{j}-f_{j}^{-1}\right)\left(f_{j}^{-N-1}-1\right)} \\
u_{n, j, 2} & =\frac{2 \omega p_{2}\left(f_{j}^{n-N-1}+f_{j}^{-n}\right)}{\pi \gamma_{2}\left(f_{j}-f_{j}^{-1}\right)\left(f_{j}^{-N-1}-1\right)}
\end{aligned}
$$


As in the conservative setting, the solution must satisfy the impact location equations:

$$
\begin{gathered}
v_{0}(0)=-p_{1} \chi_{1}(\phi)+p_{2} \chi_{2}+G(\psi)=1+a \\
v_{0}(\phi)=-p_{1} \chi_{2}+p_{2} \chi_{1}(\phi)+G(\psi+\phi)=-1+a
\end{gathered}
$$

Also, the impact law must be satisfied:

$$
\begin{gathered}
\dot{v}_{0}\left(0^{+}\right)=\dot{u}_{0}\left(0^{+}\right)+\dot{G}(\psi)= \\
=-e\left(\dot{u}_{0}\left(0^{-}\right)+\dot{G}(\psi)\right)=-e \dot{v}_{0}\left(0^{-}\right) \\
\dot{u}_{0}\left(0^{+}\right)+e \dot{u}_{0}\left(0^{-}\right)=-\dot{G}(\psi)(1+e)
\end{gathered}
$$

For the symmetric case it is clear that the energy must be conserved during each impact in terms of $u_{0}$, namely, in terms of the reduced un-forced system. Hence, for the un-forced system and the symmetric DB $\dot{u}_{0}\left(0^{+}\right)=$ $-\dot{u}_{0}\left(0^{-}\right)$and similarly for the second impact. However, this is not true for the asymmetric DB - the energy must be conserved for the reduced un-forced system (otherwise, the DB solution cannot exist), but it holds for the complete period of oscillations, and not necessarily in each single impact. So, more refined treatment is required in this case.

The generalized Fourier series converges to the average of the velocities on both sides of the discontinuity:

$$
\frac{\dot{u}_{0}\left(0^{+}\right)+\dot{u}_{0}\left(0^{-}\right)}{2}=-p_{1} \chi_{3}
$$

where

$$
\chi_{3} \equiv \sum_{j=1}^{\infty} \frac{2 j \omega^{2}\left(f_{j}^{-N-1}+1\right)}{\pi \gamma_{2}\left(f_{j}-f_{j}^{-1}\right)\left(f_{j}^{-N-1}-1\right)} \sin (j \omega \phi)
$$

Conservation of momentum during the impact yields:

$$
\frac{\dot{u}_{0}\left(0^{+}\right)-\dot{u}_{0}\left(0^{-}\right)}{2}=-p_{2}
$$

From these equations we extract terms for the velocities:

$$
\begin{gathered}
\dot{u}_{0}\left(0^{+}\right)=-p_{2}-p_{1} \chi_{3} \\
\dot{u}_{0}\left(0^{-}\right)=p_{2}-p_{1} \chi_{3}
\end{gathered}
$$

Note that the energy gain for the reduced un-forced system during the impact is $\Delta E_{1}=2 p_{1} p_{2} \chi_{3}$. Energy gain is possible since the reduced un-forced system does not represent a physical system.

Plugging into eq. (60), one obtains:

$$
\dot{G}(\psi)=p_{1} \chi_{3}+q p_{2}
$$

where $q=(1-e) /(1+e)$.
Similarly, it is possible to perform the same procedure for the second impact:

$$
\dot{u}_{0}\left(\phi^{+}\right)+e \dot{u}\left(\phi^{-}\right)=-\dot{G}(\phi+\psi)(1+e)
$$

The generalized Fourier series converges to the average of the velocities on both sides of the discontinuity:

$$
\frac{\dot{u}\left(\phi^{+}\right)+\dot{u}\left(\phi^{-}\right)}{2}=-p_{2} \chi_{3}
$$

Conservation of momentum during the impact yields:

$$
\frac{\dot{u}\left(\phi^{+}\right)-\dot{u}\left(\phi^{-}\right)}{2}=p_{1}
$$

From these equations we extract the terms for the velocities:

$$
\begin{gathered}
\dot{u}\left(\phi^{+}\right)=p_{1}-p_{2} \chi_{3} \\
\dot{u}\left(\phi^{-}\right)=-p_{1}-p_{2} \chi_{3}
\end{gathered}
$$

Note that the energy loss in this impact is $\Delta E_{2}=$ $-2 p_{1} p_{2} \chi_{3}$; hence the energy of the reduced un-forced system is conserved throughout the period as expected.

Plugging into eq. (67), one obtains:

$$
\dot{G}(\phi+\psi)=p_{2} \chi_{3}-q p_{1}
$$

\section{Harmonic Excitation}

In order to solve the equations we need to choose the forcing function, that satisfies the symmetry conditions. For simplicity, let us choose $F(t)=A \cos (\omega t)$. Solving the ODE, we obtain:

$$
G(t)=\tilde{A} \cos (\omega t)
$$

where,

$$
\tilde{A}=\frac{A}{\gamma_{1}-\omega^{2}}
$$

Plugging the solution into eq. (57), 588, 66) and (72), one obtains the following expressions:

$$
\begin{gathered}
-p_{1} \chi_{1}(\phi)+p_{2} \chi_{2}+\tilde{A} \cos (\omega \psi)=1+a \\
-p_{1} \chi_{2}+p_{2} \chi_{1}(\phi)+\tilde{A} \cos (\omega(\psi+\phi))=-1+a \\
-\tilde{A} \omega \sin (\omega(\psi+\phi))=p_{2} \chi_{3}-q p_{1} \\
-\tilde{A} \omega \sin (\omega \psi)=p_{1} \chi_{3}+q p_{2}
\end{gathered}
$$


To find the exact solution explicitly, we assume that $\phi$ is known and the barrier asymmetry $a$ is the unknown. Solution of the above set of equations under this assumption yields:

$$
\psi=\frac{ \pm \arccos \left(\frac{2\left(q^{2}+\chi_{3}^{2}\right)}{\sigma \tilde{A}}\right)+\alpha}{\omega}
$$

$$
\begin{array}{r}
p_{1}=\frac{\tilde{A} \omega\left(q \sin (\omega(\psi+\phi))-\chi_{3} \sin (\omega \psi)\right)}{q^{2}+\chi_{3}^{2}} \\
p 2=-\frac{\tilde{A} \omega\left(\chi_{3} \sin (\omega(\psi+\phi))+q \sin (\omega \psi)\right)}{q^{2}+\chi_{3}^{2}} \\
a=-p_{1} \chi_{1}(\phi)+p_{2} \chi_{2}+\tilde{A} \cos (\omega \psi)-1
\end{array}
$$

where,

$$
\begin{gathered}
\sigma=\sqrt{\begin{array}{r}
2 \omega^{2} \chi_{3}^{2}\left(\chi_{1}-\chi_{2}\right)^{2}(1+\cos (\omega \phi))+4 \omega \chi_{3}\left(q^{2}+\chi_{3}^{2}\right)\left(\chi_{1}-\chi_{2}\right) \sin (\omega \phi)+ \\
+2\left(q^{4}+\chi_{3}^{4}+q^{2}\left(\omega^{2}\left(\chi_{1}-\chi_{2}\right)^{2}+2 \chi^{3}\right)\right)(1-\cos (\omega \phi))
\end{array}} \\
\alpha= \pm \arccos \left(\frac{\left(q^{2}+\chi_{3}^{2}\right)(1-\cos (\omega \phi))-\left(q-\chi_{3}\right)\left(\chi_{1}-\chi_{2}\right) \omega \sin (\omega \phi)}{\sigma}\right)
\end{gathered}
$$

\section{Single-Sided Forced-Damped Discrete Breathers}

The single-sided DB is also possible in the forceddamped model. The equations of motion can be written as follows:

$$
\begin{gathered}
\ddot{v}_{0}+\gamma_{1} v_{0}+\gamma_{2}\left(2 v_{0}-v_{1}-v_{N}\right)= \\
=F(t+\psi)+2 p \sum_{j=-\infty}^{\infty} \delta\left(t-\frac{2 \pi j}{\omega}\right) \\
\ddot{v}_{n}+\gamma_{1} v_{n}+\gamma_{2}\left(2 v_{n}-v_{n+1}-v_{n-1}\right)=F(t+\psi) \\
\ddot{v}_{N}+\gamma_{1} v_{N}+\gamma_{2}\left(2 v_{N}-v_{0}-v_{N-1}\right)=F(t+\psi)
\end{gathered}
$$

where $\psi$ is the phase of the external force with respect to the DB's impacts.

The external force $F(t)$ can be removed from the equations in the same manner as the previous case.

As in the conservative model, the solution must satisfy the impact location equations:

$$
v_{0}(0)=-p \chi_{2}+G(\psi)=-1+a
$$

Also, the impact law must be satisfied:

$$
\begin{gathered}
\dot{v}\left(0^{+}\right)=\dot{u}\left(0^{+}\right)+\dot{G}(\psi)=p+\dot{G}(\psi)= \\
=-e(-p+\dot{G}(\psi))=-e\left(\dot{u}\left(0^{-}\right)+\dot{G}(\psi)\right)=-e \dot{v}\left(0^{-}\right)
\end{gathered}
$$

By further simplification, one obtains:

$$
\dot{G}(\psi)=-q p
$$

The full derivations are given in Appendix $\mathrm{B}$
Harmonic Excitation Let $F(t)=A \cos (\omega t)$ and,

$$
G(t)=\tilde{A} \cos (\omega t)
$$

where,

$$
\tilde{A}=\frac{A}{\gamma_{1}-\omega^{2}}
$$

In a similar manner to the regular $\mathrm{DB}$, we obtain the following equations:

$$
-p \chi_{2}+\tilde{A} \cos (\omega \psi)=-1+a
$$

$$
-\omega \tilde{A} \sin (\omega \psi)=-q p
$$

Solving the equation yields:

$$
\psi=\frac{ \pm \arccos \left(\frac{q(a-1)}{\tilde{A} \sqrt{q^{2}+\omega^{2} \chi_{2}^{2}}}\right)+\alpha}{\omega}
$$

$$
p=\frac{\omega \tilde{A}}{q} \sin (\omega \psi)
$$

where,

$$
\alpha= \pm \arccos \left(\frac{q}{\sqrt{q^{2}+\omega^{2} \chi_{2}^{2}}}\right)
$$




\section{Single-Sided Forced-Damped Discrete Breathers with Period Doubling}

The stability analysis discussed in detail in the following Section shows that one of the mechanisms for the loss of stability of the single-sided forced DB is the period doubling bifurcation. Numerical investigation shows that the period doubling is reflected by a consecutive set of collisions with different exchange of momentum in the new doubled period of the DB. This type of solution can also be obtained analytically in a similar manner to that of the forced DB with minor modifications. The location of the second impact in the period is set to the same barrier as the first collision, i.e. the closer barrier, and the period of the DB is doubled. However, obtaining a solution in this manner is only possible if the frequency of the doubled period solution is in the attenuation zone of the chain for a given set of parameters. The full derivation is given in detail in Appendix C.

\section{STABILITY}

The stability of the derived DB solutions will be investigated by Floquet theory 28. The Floquet multipliers are often evaluated numerically, but, as mentioned above, the special nature of the system allows explicit construction of the monodromy matrix. Then, computation of its eigenvalues is a relatively simple computational task, and comprehensive study of the stability patterns in the space of parameters becomes possible [21]. Moreover, eigenvectors corresponding to the unstable Floquet multipliers can be easily computed and examined to gain some qualitative insight into the mechanism of the loss of stability.

The governing equations of motion can also be written in the following equivalent form:

$$
\dot{\vec{u}}=\mathrm{A} \vec{u}
$$

where $\vec{u}=\left[\begin{array}{llllll}u_{0} & \cdots & u_{N} & \dot{u}_{0} & \cdots & \dot{u}_{N}\end{array}\right]^{T}$ and:

$$
\begin{aligned}
\mathbf{A} & =\left[\begin{array}{ccccc}
0_{(N+1) \times(N+1)} & \mathrm{I}_{(N+1) \times(N+1)} \\
\tilde{\mathrm{A}}_{(N+1) \times(N+1)} & 0_{(N+1) \times(N+1)}
\end{array}\right] \\
\tilde{A} & =\left[\begin{array}{cccccc}
\gamma_{1}+2 \gamma_{2} & -\gamma_{2} & 0 & \cdots & 0 & -\gamma_{2} \\
-\gamma_{2} & \gamma_{1}+2 \gamma_{2} & -\gamma_{2} & 0 & \cdots & 0 \\
0 & -\gamma_{2} & \ddots & \ddots & \ddots & \vdots \\
\vdots & \ddots & \ddots & \gamma_{1}+2 \gamma_{2} & -\gamma_{2} & 0 \\
0 & \cdots & 0 & -\gamma_{2} & \gamma_{1}+2 \gamma_{2} & -\gamma_{2} \\
-\gamma_{2} & 0 & \cdots & 0 & -\gamma_{2} & \gamma_{1}+2 \gamma_{2}
\end{array}\right]
\end{aligned}
$$

or for the forced-damped model:

$$
\dot{\vec{v}}=\mathrm{A} \vec{v}+\vec{F}
$$

where $\vec{F}=F(t)\left[\begin{array}{lll}1 & \cdots & 1\end{array}\right]^{T}$.

From the above equation we can derive the evolution of the perturbed phase trajectory between two impacts:

$$
\begin{gathered}
\mathrm{L}_{1}=\exp (\phi \mathrm{A}) \\
\mathrm{L}_{2}=\exp \left(\left(\frac{2 \pi}{\omega}-\phi\right) \mathrm{A}\right)
\end{gathered}
$$

or for the single-sided impact:

$$
\mathrm{L}=\exp \left(\frac{2 \pi}{\omega} \mathrm{A}\right)
$$

The impacts mapping cannot simply be based on the impact law for the stability analysis, but saltation matrix must be constructed to take into account the linear perturbations of the mapping and of the flight time to the discontinuity 29. The saltation matrix for the adopted impact law obtains the following form:

$$
\mathrm{S}_{1,2}=\left[\begin{array}{ll}
\tilde{S}_{(N+1) \times(N+1)} & 0_{(N+1) \times(N+1)} \\
\hat{\mathrm{S}}_{(N+1) \times(N+1)} & \tilde{S}_{(N+1) \times(N+1)}
\end{array}\right]
$$

where,

$$
\tilde{\mathrm{S}}=\left[\begin{array}{ccccc}
-e & 0 & \cdots & \cdots & 0 \\
0 & 1 & 0 & & \vdots \\
\vdots & 0 & \ddots & \ddots & \vdots \\
\vdots & & \ddots & 1 & 0 \\
0 & \cdots & \cdots & 0 & 1
\end{array}\right]
$$




$$
\hat{\mathrm{S}}_{1,2}=\left[\begin{array}{ccccc}
\frac{(1+e) \Delta_{1,2}}{\Gamma_{1,2}} & 0 & \cdots & \cdots & 0 \\
0 & 0 & 0 & & \vdots \\
\vdots & 0 & \ddots & \ddots & \vdots \\
\vdots & & \ddots & 0 & 0 \\
0 & \cdots & \cdots & 0 & 0
\end{array}\right]
$$

where $\Delta_{1}=\ddot{u}_{0}(\phi-), \Delta_{2}=\ddot{u}_{0}(0-),, \Gamma_{1}=-p_{1}, \Gamma_{2}=p_{2}$ for the conservative model; $\Delta_{1}=\ddot{u}_{0}(\phi-), \Delta_{2}=\ddot{u}_{0}(0-)$, , $\Gamma_{1}=-p_{1}-p_{2} \chi_{3}+\dot{G}(\phi+\psi), \Gamma_{2}=p_{2}-p_{1} \chi_{3}+\dot{G}(\psi)$ for the forced-damped model. Similarly for the Single-sided $\mathrm{DB}-\Delta_{1}=\ddot{u}_{0}(0-)$ and $\Gamma_{1}=-p$ for the conservative model; $\Delta_{1}=\ddot{u}_{0}(0-), \Gamma_{1}=-p+\dot{G}(\psi)$ for the forceddamped model. Note that for the conservative model the coefficient of restitution $e$ is set to unity.

The Monodromy matrix can be written compactly as follows:

$$
\mathrm{M}=\mathrm{L}_{1} \mathrm{~S}_{1} \mathrm{~L}_{2} \mathrm{~S}_{2}
$$

or for the single-sided DB:

$$
\mathrm{M}=\mathrm{LS}_{1}
$$

Then, the stability of the DB solution is assessed just by easy computation of this Monodromy matrix and evaluation of its spectrum.

\section{NUMERICAL VALIDATION AND STABILITY PATTERNS}

\section{A. Conservative Model}

In order to qualitatively examine the properties of the asymmetric DBs, and to validate the accuracy of our results, we turn to numerical methods. The simulations in this section are performed using MatLab. The vibroimpact response was modeled according to the impact law using event-driven algorithm. The numerical results were in agreement with the analytical results, as one should expect for the exact solutions. Thus, one can see these results as illustrations. Unless otherwise stated, the simulations were done for the following set of parameters:

$$
\begin{gathered}
\gamma_{1}=0.2 \quad \gamma_{2}=0.1 \omega=1.5 \\
N=20 \quad a=0.4
\end{gathered}
$$

As was the case in the symmetric DB, the oscillatory profile is qualitatively the same when the length of the chain is modified, as shown in fig. 1. This result conforms to the strong localization of the DB solution.

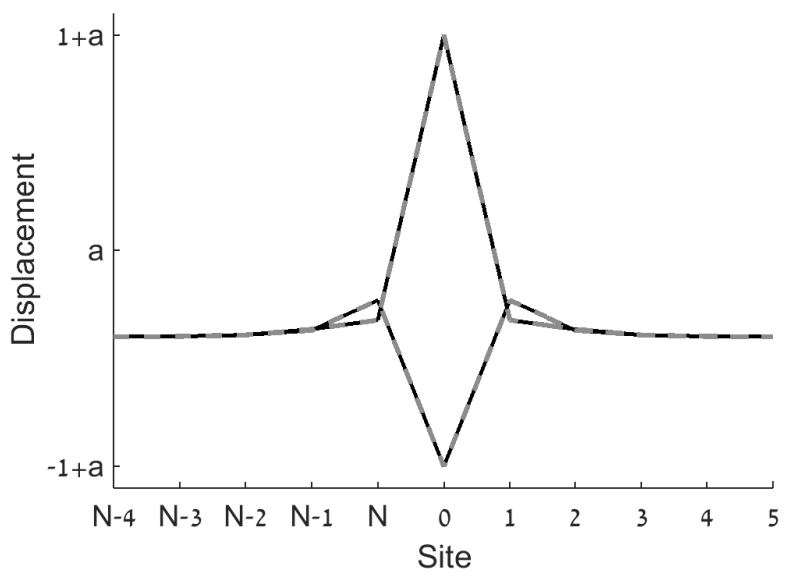

FIG. 1. The displacements of the masses for a DB solution at the instances of the two impacts for $N=10$ (Black) and $N=100$ (Dashed gray).

Figure 2 shows the strong effect of the asymmetry parameter $a$ on the DB shape. However, when examining the displacement of the impacting mass throughout the period of the DB, the difference is only a small change in the curvature as demonstrated in fig. 3. It appears that for the conservative DB, the asymmetry mainly contributes to the value to which the DB converges apart from the localization site.

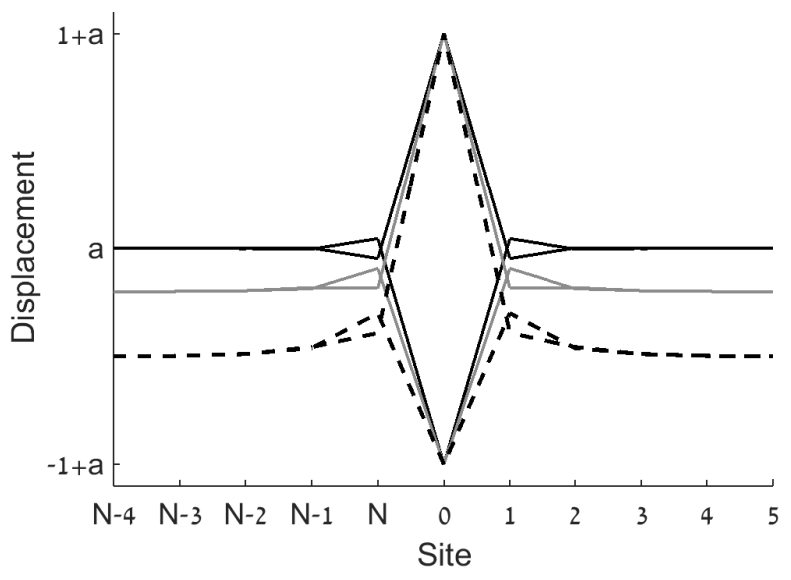

FIG. 2. The displacements of the masses for a DB solution at the instances of the two impacts for $a=0$ (Black), $a=0.2$ (Gray) and $a=0.5$ (Dashed Black). 


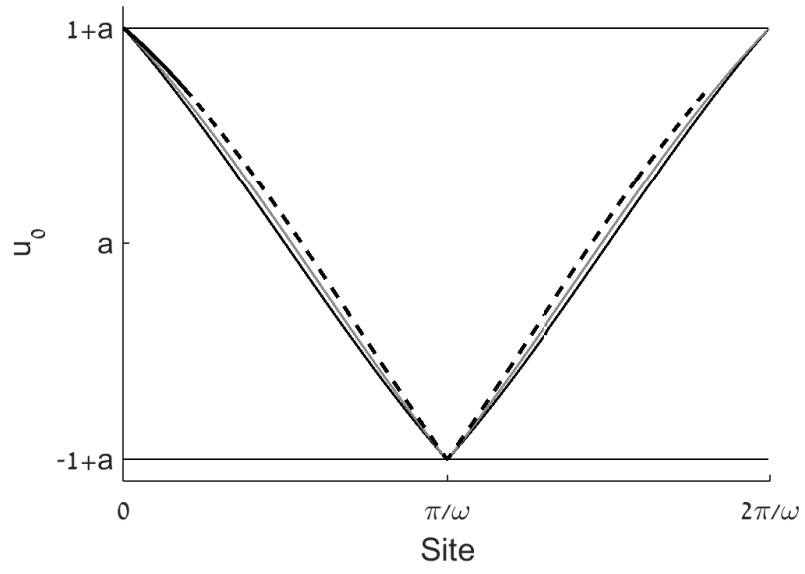

FIG. 3. The displacements of the first mass for a DB solution for $a=0$ (Black), $a=0.2$ (Gray) and $a=0.5$ (Dashed Black).

Another type of DB enabled by the asymmetry of the system is the single-sided DB. Figure 4 presents the example of the single-sided DB; note that the impacting mass does not reach the more distant barrier at $(1+a)$.

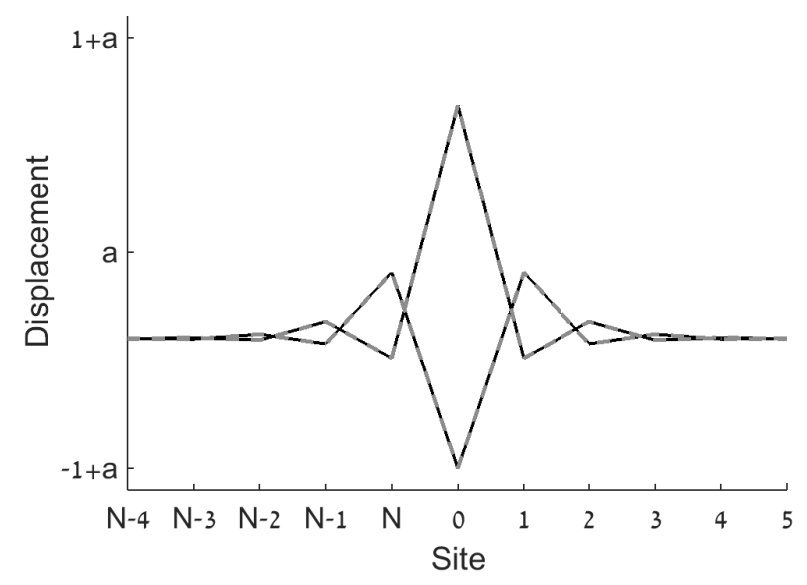

FIG. 4. The displacements of the masses for a single-sided DB solution at the instances of the two impacts.

\section{B. Forced-Damped Model}

This model is a bit more complicated to examine. As mentioned in sec. IIB, we are unable to find $\phi$ without approximations with unknown error. Therefore, $\phi$ is regarded as a known parameter and instead we obtain the asymmetry parameter $a$. Fortunately, numerical investigation reveals that the relation between $a$ and $\phi$ behaves in a manner that allows finding the wanted value of $a$ by means of iterative extrapolation with the maximal error of our choice. For time consumption purposes, the allowed error in $a$ was taken to be $10^{-12}$. Furthermore, unless stated otherwise, the parameters are as follows:

$$
\begin{gathered}
\gamma_{1}=0.2 \quad \gamma_{2}=0.1 \quad \omega=1.5 \quad N=20 \\
A=0.1 \quad e=0.9 \quad a=0.4
\end{gathered}
$$

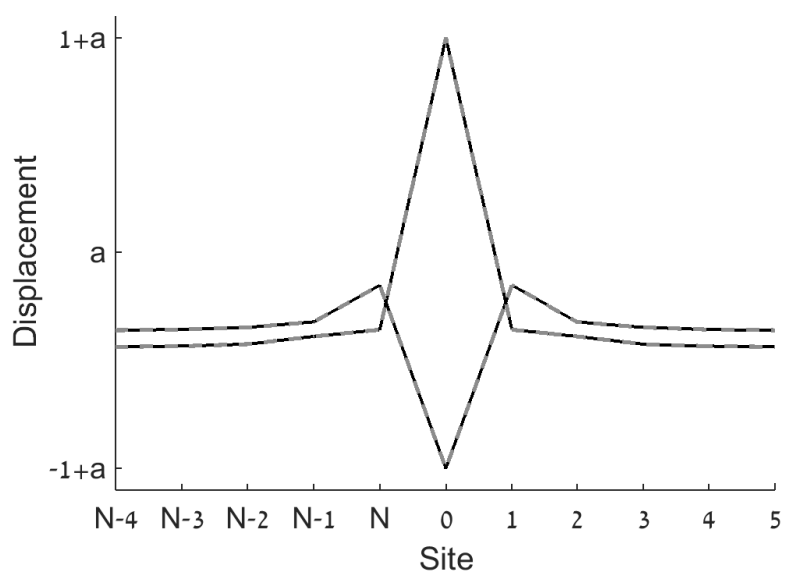

FIG. 5. The displacements of the masses for the forced DB solution at the instances of the two impacts for $N=10$ (Black) and $N=100$ (Dashed gray).

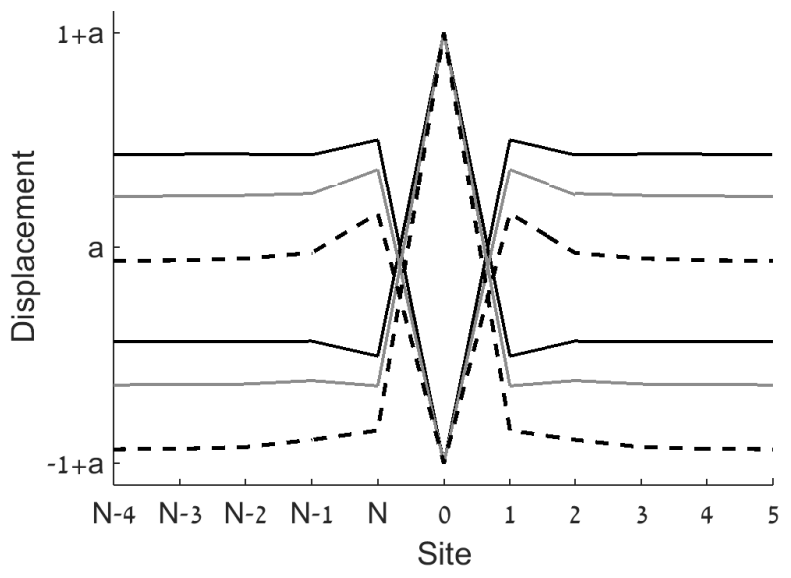

FIG. 6. The displacements of the masses for the forced DB solution at the instances of the two impacts for $A=0.9$ and $a=0$ (Black), $a=0.2$ (Gray) and $a=0.4$ (Dashed Black).

In general, the effect of the asymmetry in the forceddamped model is similar to one observed in the conservative model. In fig. 5 we see that there is no notable change in the DB profile as a result of adding masses to the chain. Figure 6 shows that, while the shape is generally different since the oscillating term converges to $G(t)$ and not to zero, as the mass is farther away from the localization site, the most profound consequence of the asymmetry is still the shift of the center of oscillations. 


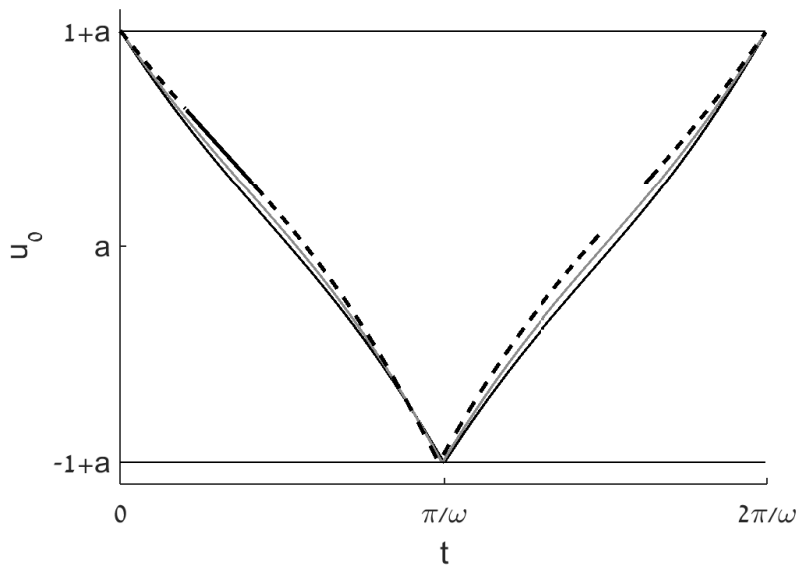

FIG. 7. The displacements of the first mass for $A=0.9$ and $a=0$ (Black), $a=0.2$ (Gray) and $a=0.5$ (Dashed Black).

Figure 7 demonstrates a main difference from the conservative DB; it clearly shows that there is a shift of the second impact, i.e. $\phi$ is diverted from $\pi / \omega$. Numerical investigation shows this difference is typically very small until the appearance of multiple solutions mentioned below.

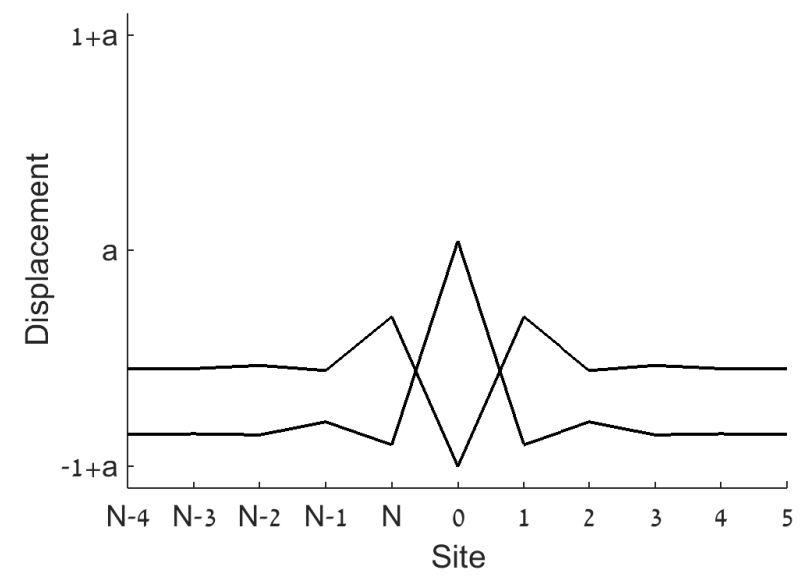

FIG. 8. The displacements of the masses for the forced singlesided DB solution at the instances of the two impacts for $\omega=0.92$.

Just like in the conservative model, the forced single- sided DBs exist as well. An example is presented in fig. 8.

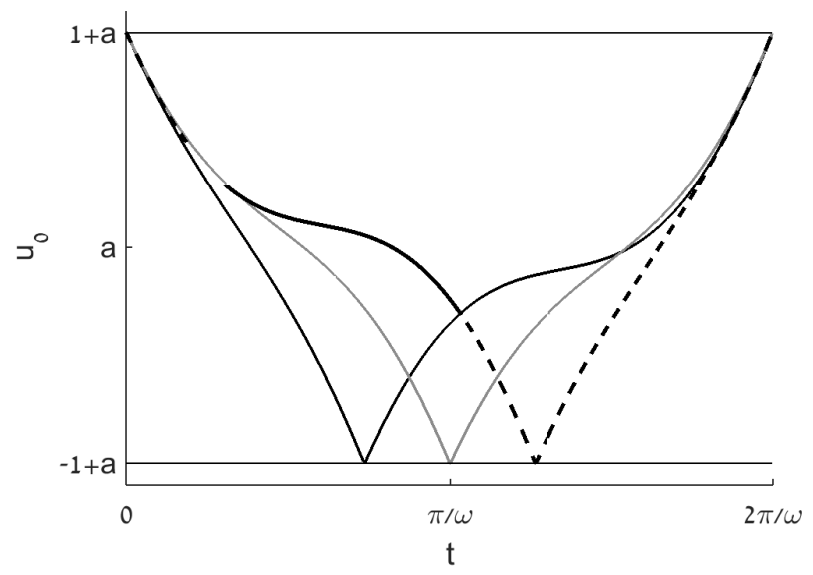

FIG. 9. The displacements of the first mass for some of the solutions for $\gamma_{1}=0.1, \gamma_{2}=0.05, \omega=1.33, A=1.5$ and $a=0$.

An interesting phenomenon appearing for stronger external forcing, i.e. larger values of $A$, is a multitude of solutions. For certain sets of parameters the analytic solution yields more than a single solution. Figure 9 shows that these solutions can even be very different from each other. It is interesting to note that it is possible that more than one of these solutions are stable.

Another interesting fact is that even for a symmetric model, namely $a=0$, an asymmetric solution could exist as predicted by Grinberg and Gendelman [22. one or more of these solutions appear to become stable when the symmetric DB losses stability via the pitchfork bifurcation.

\section{Stability}

The procedure for the stability analysis is described in detail in sec. III. Additionally, the following stability maps also refer to existence of the solution, i.e. solutions that are not physical, e.g. some masses exceed the boundaries, are marked as non-existent. The set of parameters is similar to that in the previous sub-sections, unless stated otherwise.

We begin with investigation of the forced-damped model; since the stable solutions of the forced-damped model generically are hyperbolic attractors, the numeric validation of the stability analysis is easier than in the conservative model. 


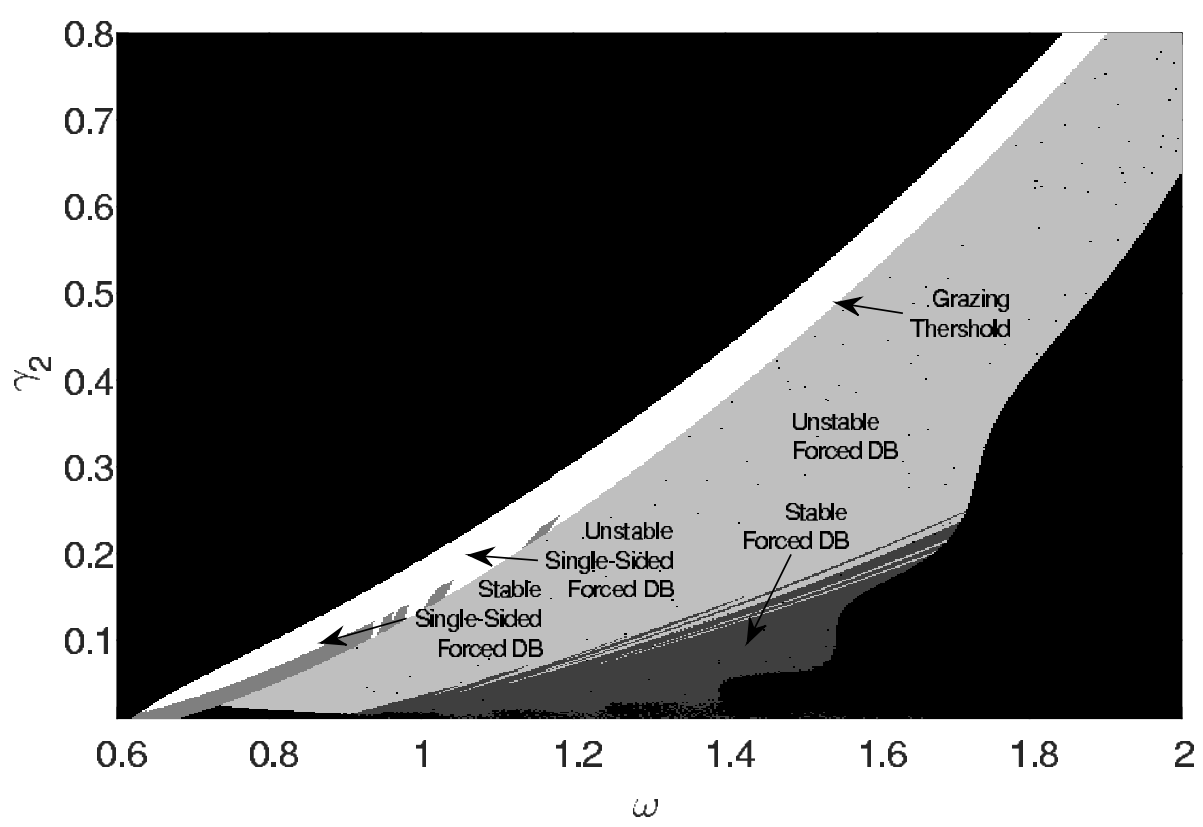

FIG. 10. Existance-stability map for the forced-damped DB. The map shows non-existing or not-physical solutions (black), stable forced DB (dark gray), stable single-sided forced DB (gray), unstable forced DB (light gray), and unstable single-sided forced DB (white)

Figure 10 shows the region of stability and existence of the forced-damped DB in the frequency - coupling stiffness plane of parameters. In this map the mechanism for loss of stability for both double- and single-sided forced DBs is Neimark-Sacker bifurcation; however, other mechanisms for the loss of stability appear for other sets of parameters - the period doubling and pitchfork bifurcations are also encountered. One can also clearly see the grazing threshold where the forced double-sided DB surpasses the grazing point and turns into the single-sided DB.

It is important to note that at the grazing limit, the stable solution of the single-sided forced DB meets the unstable forced DB. The convergence of the stability and grazing boundaries can be explained by the sudden breaking of symmetry of the solution. While the singlesided forced DB is symmetric with respect to the peak between two impacts, after reaching the grazing point and with the transition to the forced DB the second impact in the period immediately diverges from $\phi=\pi / w$, where it occurs at the grazing point.

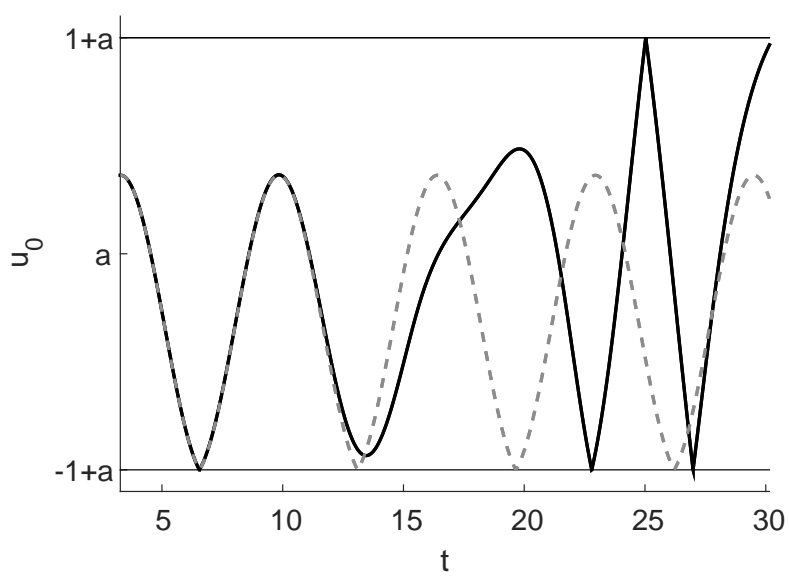

FIG. 11. The displacements of the first mass (Numerical result in black and analytic solution in dashed gray) for an unstable single-sided forced DB via Neimark-Sacker bifurcation. 


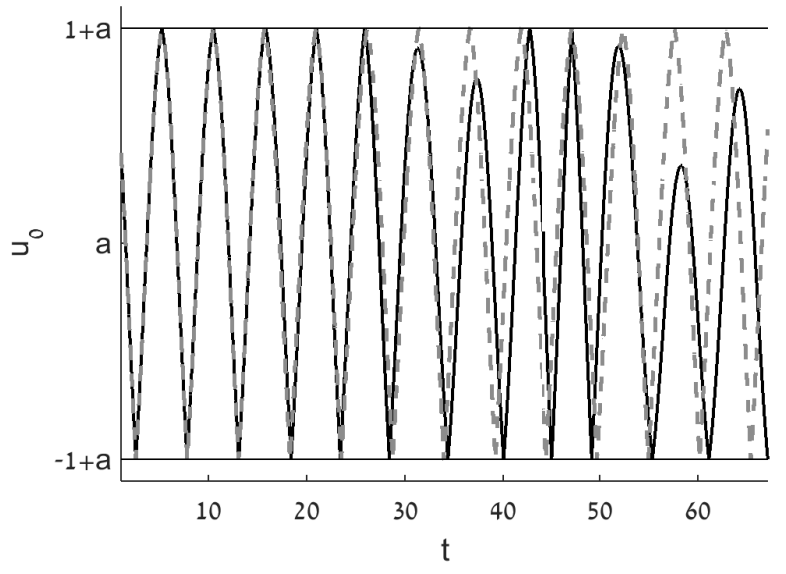

FIG. 12. The displacements of the first mass (Numerical result in black and analytic solution in dashed gray) for an unstable forced DB via Neimark-Sacker bifurcation.

Figures 11 and 12 present examples of the loss of stability via Neimark-Sacker bifurcation. The discrepancy between the numerical solution and analytic prediction for the unstable solution validates the procedure of the stability analysis presented above.

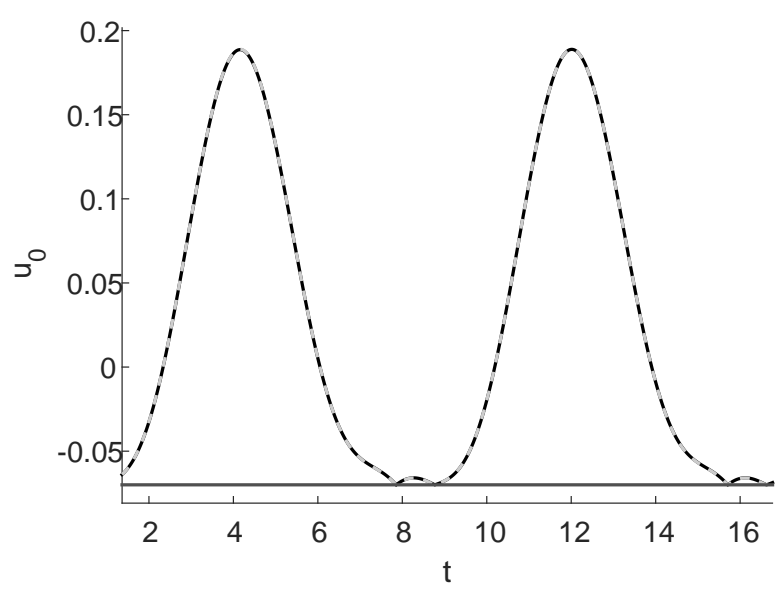

FIG. 13. The displacements of the first mass (Numerical result in black and analytic solution in dashed gray) for the forced single-sided DB with period doubling.

As aforementioned, there are two more mechanisms for the loss of stability. The first is the pitchfork bifurcation of the forced DB; pair of stable asymmetric solutions is formed. These asymmetric solutions were predicted numerically in ref. 22 and found analytically in this work. The last mechanism for loss of stability is through period doubling bifurcation relevant to the single-sided forced DB. The period doubling obtained analytically is demonstrated in fig. 13. The period doubling is clearly visible in the form to two consecutive peaks of different heights in each period. 

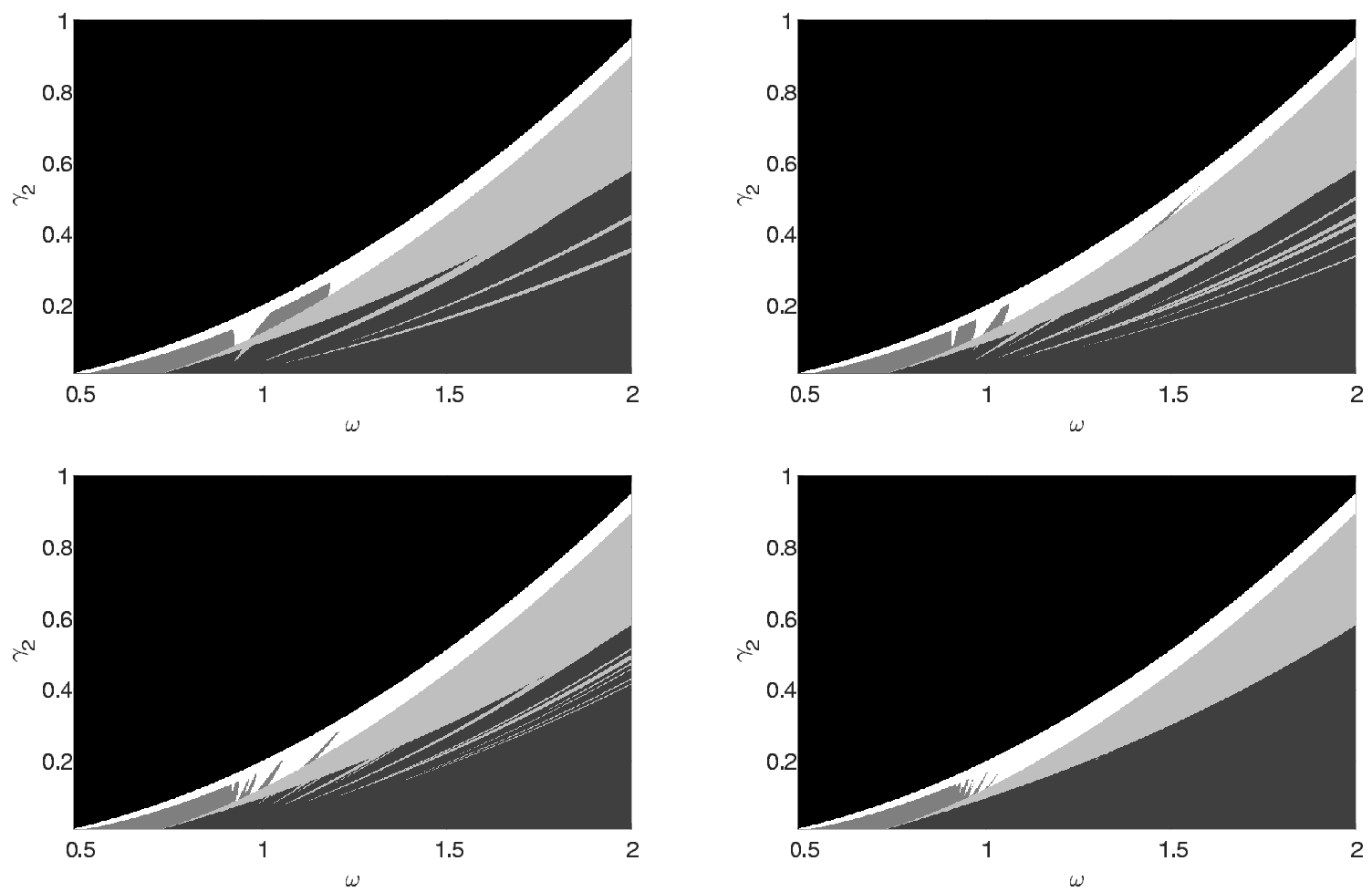

FIG. 14. Existence-stability maps for a DB with $N=5, N=10, N=20$ and $N=40$ (Top to Bottom). The map shows non-existing or not-physical solutions (black), stable DB (dark gray), stable single-sided DB (gray), unstable DB (light gray), and unstable single-sided DB (white)

Figure 14 shows the existence-stability map for the conservative model. There is a lot of similarity in this map to that of the forced DB. The loss of stability here as well is via Neimark-Sacker bifurcation. However, in the case of the conservative system it is difficult to validate the loss of stability, since its solutions are not attractors. Other similar features are the stripes of instability. These stripes are related to the chain length as demonstrated in the existence-stability maps for different chain length in fig. 14, and correspond to the spatial modes appearing in the eigenvectors of the Monodromy matrix due to the finite size of the system, resembling the observations in ref. [22]. It is also interesting to note that, with the exception of these stripes, the stability patterns are not strongly affected by the system size.

\section{CONCLUDING REMARKS}

In this work, we derive the exact DB solutions for asymmetric vibro-impact lattice. Contrary to the symmetric setting, two types of such solutions exist - the single-sided and the double-sided DBs, divided by the grazing boundary. The asymmetric DBs can appear not only in the intrinsically asymmetric lattice considered in the paper, but also can result from the symmetry braking in the symmetric lattices investigated in previous works.

Another interesting finding is the multiplicity of the stable forced DB solutions, not observed in the symmetric lattice. It is possible to observe more than one stable asymmetric solution for the same set of parameters. The finite system size leads to formation of the "stripes of instability", which appear due to the finite set of eigenmodes available in the finite system. This peculiarity essentially modifies the domain of stability on the space of parameters and can be considered as generic consequence of the finite system size.

Finally, the asymmetric setting reveal all three generic mechanisms for loss of stability of the periodic solutions - pitchfork, Neimark-Sacker and period doubling bifurcations. The latter was not observed in previously studied symmetric models and an exact analytic solution for the doubled period solutions is also obtained.

\section{ACKNOWLEDGMENTS}

The authors are very grateful to Israel Science Foundation (grant 838/13) for financial support. 
[1] S. Flach and C.R. Willis. Discrete breathers. Physics Reports, 295(5):181 - 264, 1998.

[2] Sergej Flach and Andrey V. Gorbach. Discrete breathers - advances in theory and applications. Physics Reports, 467(1-3):1 - 116, 2008.

[3] Alexander F Vakakis, Leonid I Manevitch, Yuri V Mikhlin, Valery N Pilipchuk, and Alexandr A Zevin. Normal modes and localization in nonlinear systems. Springer, 1996.

[4] David K Campbell. Nonlinear physics: Fresh breather. Nature, 432(7016):455-456, 2004.

[5] Alexander F Vakakis, Oleg V Gendelman, Lawrence A Bergman, D Michael McFarland, Gaëtan Kerschen, and Young Sup Lee. Nonlinear targeted energy transfer in mechanical and structural systems, volume 156. Springer Science \& Business Media, 2008.

[6] P. W. Anderson. Absence of diffusion in certain random lattices. Phys. Rev., 109:1492-1505, Mar 1958.

[7] C. Pierre and E.H. Dowell. Localization of vibrations by structural irregularity. Journal of Sound and Vibration, 114(3):549 - 564, 1987.

[8] O.O. Bendiksen. Localization phenomena in structural dynamics. Chaos, Solitons \& Fractals, 11(10):1621 1660, 2000.

[9] David Cai, A.R. Bishop, Niels Grønbech-Jensen, and Boris A. Malomed. Moving solitons in the damped ablowitz-ladik model driven by a standing wave. Phys. Rev. E, 50:R694-R697, Aug 1994.

[10] E. Trías, J. J. Mazo, and T. P. Orlando. Discrete breathers in nonlinear lattices: Experimental detection in a josephson array. Phys. Rev. Lett., 84:741-744, Jan 2000 .

[11] N. Lazarides, M. Eleftheriou, and G. P. Tsironis. Discrete breathers in nonlinear magnetic metamaterials. Phys. Rev. Lett., 97:157406, Oct 2006.

[12] L. Q. English, F. Palmero, P. Candiani, J. Cuevas, R. Carretero-González, P. G. Kevrekidis, and A. J. Sievers. Generation of localized modes in an electrical lattice using subharmonic driving. Phys. Rev. Lett., 108:084101, Feb 2012.

[13] S. Gutschmidt and O. Gottlieb. Bifurcations and loss of orbital stability in nonlinear viscoelastic beam arrays subject to parametric actuation. Journal of Sound and Vibration, 329(18):3835 - 3855, 2010.

[14] Masayuki Kimura and Takashi Hikihara. Coupled cantilever array with tunable on-site nonlinearity and observation of localized oscillations. Physics Letters A, 373(14):1257 - 1260, 2009.

[15] Eyal Kenig, Boris A. Malomed, M. C. Cross, and Ron Lifshitz. Intrinsic localized modes in parametrically driven arrays of nonlinear resonators. Phys. Rev. E, 80:046202, Oct 2009.

[16] M. Sato, S. Imai, N. Fujita, S. Nishimura, Y. Takao, Y. Sada, B. E. Hubbard, B. Ilic, and A. J. Sievers. Experimental observation of the bifurcation dynamics of an intrinsic localized mode in a driven $1 \mathrm{~d}$ nonlinear lattice. Phys. Rev. Lett., 107:234101, Nov 2011.

[17] M. Sato, B. E. Hubbard, and A. J. Sievers. Colloquium : Nonlinear energy localization and its manipulation in micromechanical oscillator arrays. Rev. Mod. Phys., 78:137157, Jan 2006.
[18] Andrea Trombettoni and Augusto Smerzi. Discrete solitons and breathers with dilute bose-einstein condensates. Phys. Rev. Lett., 86:2353-2356, Mar 2001.

[19] O. V. Gendelman and L. I. Manevitch. Discrete breathers in vibroimpact chains: Analytic solutions. Phys. Rev. E, 78:026609, Aug 2008.

[20] J. Cuevas, L. Q. English, P. G. Kevrekidis, and M. Anderson. Discrete breathers in a forced-damped array of coupled pendula: Modeling, computation, and experiment. Phys. Rev. Lett., 102:224101, Jun 2009.

[21] O. V. Gendelman. Exact solutions for discrete breathers in a forced-damped chain. Phys. Rev. E, 87:062911, Jun 2013.

[22] Itay Grinberg and Oleg V. Gendelman. Localization in finite vibroimpact chains: Discrete breathers and multibreathers. Phys. Rev. E, 94:032204, Sep 2016.

[23] Nathan Perchikov and O.V. Gendelman. Dynamics and stability of a discrete breather in a harmonically excited chain with vibro-impact on-site potential. Physica D: Nonlinear Phenomena, 292-293:8 - 28, 2015.

[24] F. Romeo and O.V. Gendelman. Discrete breathers in forced chains of oscillators with cubic nonlinearities. Procedia IUTAM, 19:236-243, 2016.

[25] M. J. Ablowitz and J. F. Ladik. Nonlinear differentialdifference equations and fourier analysis. Journal of Mathematical Physics, 17(6):1011-1018, 1976.

[26] A. A. Ovchinnikov and S. Flach. Discrete breathers in systems with homogeneous potentials: Analytic solutions. Phys. Rev. Lett., 83:248-251, Jul 1999.

[27] E.B. Shiroky and O.V. Gendelman. Discrete breathers in an array of self-excited oscillators: Exact solutions and stability. Chaos, 26:103112, Oct 2016.

[28] S.H. Strogatz. Nonlinear Dynamics and Chaos: With Applications to Physics, Biology, Chemistry, and Engineering. Advanced book program. Westview Press, 1994.

[29] Mats H. Fredriksson and Arne B. Nordmark. On normal form calculations in impact oscillators. Proceedings of the Royal Society of London A: Mathematical, Physical and Engineering Sciences, 456(1994):315-329, 2000.

\section{Appendix A: Single-Sided Discrete Breather - Derivations}

The governing equations of motion are:

$$
\begin{gathered}
\ddot{u}_{0}+\gamma_{1} u_{0}+\gamma_{2}\left(2 u_{0}-u_{1}-u_{N}\right)=2 p \sum_{j=-\infty}^{\infty} \delta\left(t-\frac{2 \pi j}{\omega}\right) \\
\ddot{u}_{n}+\gamma_{1} u_{n}+\gamma_{2}\left(2 u_{n}-u_{n+1}-u_{n-1}\right)=0 \\
\ddot{u}_{N}+\gamma_{1} u_{N}+\gamma_{2}\left(2 u_{N}-u_{0}-u_{N-1}\right)=0
\end{gathered}
$$

The terms for the impacts can also be written in the form of generalized Fourier series:

$$
\ddot{u}_{0}+\gamma_{1} u_{0}+\gamma_{2}\left(2 u_{0}-u_{1}-u_{N}\right)=\frac{\omega p}{\pi} \sum_{j=-\infty}^{\infty} \cos (j \omega t)
$$




$$
\begin{aligned}
& \ddot{u}_{n}+\gamma_{1} u_{n}+\gamma_{2}\left(2 u_{n}-u_{n+1}-u_{n-1}\right)=0 \\
& \ddot{u}_{N}+\gamma_{1} u_{N}+\gamma_{2}\left(2 u_{N}-u_{0}-u_{N-1}\right)=0
\end{aligned}
$$

This can be rewritten as follows:

$$
\begin{array}{r}
\ddot{u}_{0}+\gamma_{1} u_{0}+\gamma_{2}\left(2 u_{0}-u_{1}-u_{N}\right)=\frac{\omega}{\pi} p+\frac{2 \omega p}{\pi} \sum_{j=1}^{\infty} \cos (j \omega t) \\
\ddot{u}_{n}+\gamma_{1} u_{n}+\gamma_{2}\left(2 u_{n}-u_{n+1}-u_{n-1}\right)=0 \\
\ddot{u}_{N}+\gamma_{1} u_{N}+\gamma_{2}\left(2 u_{N}-u_{0}-u_{N-1}\right)=0 \\
\text { (A8) }
\end{array}
$$

Solving in a similar manner, we obtain:

$$
u_{n}=u_{n, 0}+\sum_{j=1}^{\infty} u_{n, j} \cos (j \omega t)
$$

where,

$$
\begin{aligned}
& u_{n, 0}=-\frac{\omega p\left(f_{0}^{n-N-1}+f_{0}^{-n}\right)}{\pi \gamma_{2}\left(f_{0}-f_{0}^{-1}\right)\left(f_{0}^{-N-1}-1\right)} \\
& u_{n, j}=-\frac{2 \omega p\left(f_{j}^{n-N-1}+f_{j}^{-n}\right)}{\pi \gamma_{2}\left(f_{j}-f_{j}^{-1}\right)\left(f_{j}^{-N-1}-1\right)}
\end{aligned}
$$

As previously, the system is closed with the help of equations, that fix the impact at the desired location:

$$
\begin{gathered}
u_{0}(0)=-\frac{\omega\left(f_{0}^{-N-1}+1_{0}\right) p}{\pi \gamma_{2}\left(f_{0}-f_{0}^{-1}\right)\left(f_{0}^{-N-1}-1\right)}- \\
-\sum_{j=1}^{\infty} \frac{2 \omega\left(f_{j}^{-N-1}+1\right) p}{\pi \gamma_{2}\left(f_{j}-f_{j}^{-1}\right)\left(f_{j}^{-N-1}-1\right)}=-1+a \\
-p \chi_{2}=-1+a \rightarrow p=\frac{1-a}{\chi_{2}}
\end{gathered}
$$

\section{Appendix B: Single-Sided Forced-Damped Discrete Breather - Derivations}

The single-sided DB is also possible in the forceddamped model. The equations of motion can be written as follows:

$$
\begin{gathered}
\ddot{v}_{0}+\gamma_{1} v_{0}+\gamma_{2}\left(2 v_{0}-v_{1}-v_{N}\right)= \\
=F(t+\psi)+2 p \sum_{j=-\infty}^{\infty} \delta\left(t-\frac{2 \pi j}{\omega}\right) \\
\ddot{v}_{n}+\gamma_{1} v_{n}+\gamma_{2}\left(2 v_{n}-v_{n+1}-v_{n-1}\right)=F(t+\psi) \\
\ddot{v}_{N}+\gamma_{1} v_{N}+\gamma_{2}\left(2 v_{N}-v_{0}-v_{N-1}\right)=F(t+\psi)
\end{gathered}
$$

where $\psi$ is the phase external force with respect to DB's impacts.
The external force $F(t)$ can be removed from the equations with the help of a simple transformation. Let $v_{n}(t)=u_{n}(t)+G(t+\psi)$ where $\ddot{G}(t)+\gamma_{1} G(t)=F(t)$. Substitution into the above equations yields:

$$
\begin{gathered}
\ddot{u}_{0}+\gamma_{1} u_{0}+\gamma_{2}\left(2 u_{0}-u_{1}-u_{N}\right)=2 p \sum_{j=-\infty}^{\infty} \delta\left(t-\frac{2 \pi j}{\omega}\right) \\
\ddot{u}_{n}+\gamma_{1} u_{n}+\gamma_{2}\left(2 u_{n}-u_{n+1}-u_{n-1}\right)=0 \\
\ddot{u}_{N}+\gamma_{1} u_{N}+\gamma_{2}\left(2 u_{N}-u_{0}-u_{N-1}\right)=0
\end{gathered}
$$

Similarly, the terms for the impacts can is written in the form of generalized Fourier series:

$$
\begin{gathered}
\ddot{u}_{0}+\gamma_{1} u_{0}+\gamma_{2}\left(2 u_{0}-u_{1}-u_{N}\right)=\frac{\omega p}{\pi} \sum_{j=-\infty}^{\infty} \cos (j \omega t) \\
\ddot{u}_{n}+\gamma_{1} u_{n}+\gamma_{2}\left(2 u_{n}-u_{n+1}-u_{n-1}\right)=0 \\
\ddot{u}_{N}+\gamma_{1} u_{N}+\gamma_{2}\left(2 u_{N}-u_{0}-u_{N-1}\right)=0
\end{gathered}
$$

Since the equations are identical to those of the conservative model, the solution is similar:

$$
u_{n}=u_{n, 0}+\sum_{j=1}^{\infty}\left(u_{n, j} \cos (j \omega t)\right)
$$

where,

$$
\begin{aligned}
& u_{n, 0}=-\frac{\omega p\left(f_{0}^{n-N-1}+f_{0}^{-n}\right)}{\pi \gamma_{2}\left(f_{0}-f_{0}^{-1}\right)\left(f_{0}^{-N-1}-1\right)} \\
& u_{n, j}=-\frac{2 \omega p\left(f_{j}^{n-N-1}+f_{j}^{-n}\right)}{\pi \gamma_{2}\left(f_{j}-f_{j}^{-1}\right)\left(f_{j}^{-N-1}-1\right)}
\end{aligned}
$$

As in the conservative model, the solution must satisfy the impact location equations:

$$
v_{0}(0)=-p \chi_{2}+G(\psi)=-1+a
$$

Also, the impact law must be satisfied:

$$
\begin{gathered}
\dot{v}\left(0^{+}\right)=\dot{u}\left(0^{+}\right)+\dot{G}(\psi)=p+\dot{G}(\psi)= \\
=-e(-p+\dot{G}(\psi))=-e\left(\dot{u}\left(0^{-}\right)+\dot{G}(\psi)\right)=-e \dot{v}\left(0^{-}\right)
\end{gathered}
$$

By further simplification, one obtains:

$$
\dot{G}(\psi)=-q p
$$

where $q=(1-e) /(1+e)$. 


\section{Appendix C: Single-Sided Forced-Damped Discrete Breather with Period Doubling - Derivations}

We examine once more the case of a symmetric force $F(t)$ which satisfies $F(t)=F(t+2 \pi / \Omega)$ and $F(t)=$ $-F(t+\pi / \Omega)$, however, with a frequency of $\Omega=2 \omega$. The solution should obey the following set of equations:

$$
\begin{gathered}
\ddot{v}_{0}+\gamma_{1} v_{0}+\gamma_{2}\left(2 v_{0}-v_{1}-v_{N}\right)=F(t+\psi)+ \\
+2 p_{1} \sum_{j=-\infty}^{\infty} \delta\left(t-\phi-\frac{2 \pi j}{\omega}\right)- \\
+2 p_{2} \sum_{j=-\infty}^{\infty} \delta\left(t-\frac{2 \pi j}{\omega}\right) \\
\ddot{v}_{n}+\gamma_{1} v_{n}+\gamma_{2}\left(2 v_{n}-v_{n+1}-v_{n-1}\right)=F(t+\psi) \\
\ddot{v}_{N}+\gamma_{1} v_{N}+\gamma_{2}\left(2 v_{N}-v_{0}-v_{N-1}\right)=F(t+\psi)
\end{gathered}
$$

where $\psi$ is the phase of the external force with respect to DB's impacts.

The external force $F(t)$ can be removed from the equations with the help of a simple transformation. Let $v_{n}(t)=u_{n}(t)+G(t+\psi)$ where $\ddot{G}(t)+\gamma_{1} G(t)=F(t)$. Substitution into the above equations yields:

$$
\begin{gathered}
\ddot{u}_{0}+\gamma_{1} u_{0}+\gamma_{2}\left(2 u_{0}-u_{1}-u_{N}\right)= \\
=2 p_{1} \sum_{j=-\infty}^{\infty} \delta\left(t-\phi-\frac{2 \pi j}{\omega}\right)- \\
+2 p_{2} \sum_{j=-\infty}^{\infty} \delta\left(t-\frac{2 \pi j}{\omega}\right) \\
\ddot{u}_{n}+\gamma_{1} u_{n}+\gamma_{2}\left(2 u_{n}-u_{n+1}-u_{n-1}\right)=0 \\
\ddot{u}_{N}+\gamma_{1} u_{N}+\gamma_{2}\left(2 u_{N}-u_{0}-u_{N-1}\right)=0
\end{gathered}
$$

Similarly, the terms for the impacts is written in the form of generalized Fourier series:

$$
\begin{gathered}
\ddot{u}_{0}+\gamma_{1} u_{0}+\gamma_{2}\left(2 u_{0}-u_{1}-u_{N}\right)= \\
=\frac{\omega}{\pi} \sum_{j=-\infty}^{\infty}\left(p_{1} \cos (j \omega(t-\phi))+p_{2} \cos (j \omega t)\right) \\
\ddot{u}_{n}+\gamma_{1} u_{n}+\gamma_{2}\left(2 u_{n}-u_{n+1}-u_{n-1}\right)=0 \\
\ddot{u}_{N}+\gamma_{1} u_{N}+\gamma_{2}\left(2 u_{N}-u_{0}-u_{N-1}\right)=0
\end{gathered}
$$

The equations are identical to those of the conservative model. Hence, the solution is similar:

$u_{n}=u_{n, 0}+\sum_{j=1}^{\infty}\left(u_{n, j, 1} \cos (j \omega(t-\phi))+u_{n, j, 2} \cos (j \omega t)\right)$ where

$$
\begin{aligned}
u_{n, 0} & =-\frac{\omega\left(p_{2}+p_{1}\right)\left(f_{0}^{n-N-1}+f_{0}^{-n}\right)}{\pi \gamma_{2}\left(f_{0}-f_{0}^{-1}\right)\left(f_{0}^{-N-1}-1\right)} \\
u_{n, j, 1} & =-\frac{2 \omega p_{1}\left(f_{j}^{n-N-1}+f_{j}^{-n}\right)}{\pi \gamma_{2}\left(f_{j}-f_{j}^{-1}\right)\left(f_{j}^{-N-1}-1\right)} \\
u_{n, j, 2} & =-\frac{2 \omega p_{2}\left(f_{j}^{n-N-1}+f_{j}^{-n}\right)}{\pi \gamma_{2}\left(f_{j}-f_{j}^{-1}\right)\left(f_{j}^{-N-1}-1\right)}
\end{aligned}
$$

As in the conservative setting, the solution must satisfy the impact location equations:

$$
\begin{gathered}
v_{0}(0)=-p_{1} \chi_{1}(\phi)-p_{2} \chi_{2}+G(\psi)=-1+a \\
v_{0}(\phi)=-p_{1} \chi_{2}-p_{2} \chi_{1}(\phi)+G(\psi+\phi)=-1+a
\end{gathered}
$$

Also, the impact law must be satisfied:

$$
\begin{gathered}
\dot{v}_{0}\left(0^{+}\right)=\dot{u}_{0}\left(0^{+}\right)+\dot{G}(\psi)= \\
=-e\left(\dot{u}_{0}\left(0^{-}\right)+\dot{G}(\psi)\right)=-e \dot{v}_{0}\left(0^{-}\right) \\
\dot{u}_{0}\left(0^{+}\right)+e \dot{u}_{0}\left(0^{-}\right)=-\dot{G}(\psi)(1+e)
\end{gathered}
$$

The generalized Fourier series converges to the average of the velocities on both sides of the discontinuity:

$$
\frac{\dot{u}_{0}\left(0^{+}\right)+\dot{u}_{0}\left(0^{-}\right)}{2}=-p_{1} \chi_{3}
$$

Conservation of momentum during the impact yields:

$$
\frac{\dot{u}_{0}\left(0^{+}\right)-\dot{u}_{0}\left(0^{-}\right)}{2}=p_{2}
$$

From these equations we extract terms for the velocities:

$$
\begin{gathered}
\dot{u}_{0}\left(0^{+}\right)=p_{2}-p_{1} \chi_{3} \\
\dot{u}_{0}\left(0^{-}\right)=-p_{2}-p_{1} \chi_{3}
\end{gathered}
$$

Note that the change in energy for the reduced unforced system during the impact is $\Delta E_{1}=-2 p_{1} p_{2} \chi_{3}$.

Plugging into eq. C17, one obtains:

$$
\dot{G}(\psi)=p_{1} \chi_{3}-q p_{2}
$$

where $q=(1-e) /(1+e)$.

Similarly, it is possible to perform the same procedure for the second impact:

$$
\dot{u}_{0}\left(\phi^{+}\right)+e \dot{u}\left(\phi^{-}\right)=-\dot{G}(\phi+\psi)(1+e)
$$

The generalized Fourier series converges to the average of the velocities on both sides of the discontinuity: 


$$
\frac{\dot{u}\left(\phi^{+}\right)+\dot{u}\left(\phi^{-}\right)}{2}=p_{2} \chi_{3}
$$

Conservation of momentum during the impact yields:

$$
\frac{\dot{u}\left(\phi^{+}\right)-\dot{u}\left(\phi^{-}\right)}{2}=p_{1}
$$

From these equations we extract the terms for the velocities:

$$
\begin{gathered}
\dot{u}\left(\phi^{+}\right)=p_{1}+p_{2} \chi_{3} \\
\dot{u}\left(\phi^{-}\right)=-p_{1}+p_{2} \chi_{3}
\end{gathered}
$$

Note that the energy gain in this impact is $\Delta E_{2}=$ $2 p_{1} p_{2} \chi_{3}$; hence the energy of the reduced un-forced system is conserved throughout the period as expected.

Plugging into eq. C23:

$$
\dot{G}(\phi+\psi)=-p_{2} \chi_{3}-q p_{1}
$$

\section{a. Harmonic Excitation}

In order to solve the equations we need to choose the forcing function, that satisfies the symmetry conditions. Let us choose $F(t)=A \cos (2 \omega t)$. Solving the ODE, we obtain:

$$
G(t)=\tilde{A} \cos (2 \omega t)
$$

where,

$$
\tilde{A}=\frac{A}{\gamma_{1}-(2 \omega)^{2}}
$$

Plugging the solution into eq. C14, $\mathrm{C} 15, \mathrm{C} 22$ and (C28), one obtains the following expressions:

$$
\begin{gathered}
-p_{1} \chi_{1}(\phi)-p_{2} \chi_{2}+\tilde{A} \cos (2 \omega \psi)=-1+a \\
-p_{1} \chi_{2}-p_{2} \chi_{1}(\phi)+\tilde{A} \cos (2 \omega(\psi+\phi))=-1+a \\
-2 \tilde{A} \omega \sin (2 \omega(\psi+\phi))=-p_{2} \chi_{3}-q p_{1} \\
-2 \tilde{A} \omega \sin (2 \omega \psi)=p_{1} \chi_{3}-q p_{2}
\end{gathered}
$$

Note that for $\phi=\pi / \omega$ we obtain this set of equation is reduced to two independent equations and we get the regular single-sided forced DB solution.

To find the exact solution explicitly, we assume that $\phi$ is known and the barrier asymmetry $a$ is the unknown. Solution of the above set of equations under this assumption yields:

$$
\psi=\frac{ \pm \frac{\pi}{2}+\alpha}{2 \omega}
$$

$$
p_{1}=\frac{2 \tilde{A} \omega\left(q \sin (2 \omega(\psi+\phi))-\chi_{3} \sin (2 \omega \psi)\right)}{q^{2}+\chi_{3}^{2}}
$$

$$
p 2=-\frac{2 \tilde{A} \omega\left(\chi_{3} \sin (2 \omega(\psi+\phi))+q \sin (2 \omega \psi)\right)}{q^{2}+\chi_{3}^{2}}
$$

$$
a=-p_{1} \chi_{1}(\phi)-p_{2} \chi_{2}+\tilde{A} \cos (2 \omega \psi)+1
$$

where,

$$
\begin{gathered}
\sigma=\sqrt{\begin{array}{r}
8 \omega^{2} \chi_{3}^{2}\left(\chi_{1}-\chi_{2}\right)^{2}(1+\cos (2 \omega \phi))+8 \omega \chi_{3}\left(q^{2}+\chi_{3}^{2}\right)\left(\chi_{1}-\chi_{2}\right) \sin (2 \omega \phi)+ \\
+2\left(\chi_{3}^{4}-q^{4}+q^{2}\left(4 \omega^{2}\left(\chi_{1}-\chi_{2}\right)^{2}+2 \chi^{3}\right)\right)(1-\cos (2 \omega \phi))
\end{array}} \\
\alpha= \pm \arccos \left(\frac{\left(q^{2}+\chi_{3}^{2}\right)(1-\cos (2 \omega \phi))-\left(q-\chi_{3}\right)\left(\chi_{1}-\chi_{2}\right) \omega \sin (2 \omega \phi)}{\sigma}\right)
\end{gathered}
$$

